\title{
Educación y algunas políticas educativas
}

Por: Cindy Paola Cristancho Sanabria ${ }^{1}$

pecas88@gmail.com

\section{La educación: a manera de contextualización.}

La educación sin duda alguna se ha constituido como una práctica y acto social trascendental, en la medida que a través de diversidad de medios (implícitos o explícitos) se convierte en un poderoso medio de socialización (Durkheim, 1970), ésta entendida como el proceso de inserción de los individuos desde el mismo momento de su nacimiento a la dinámica de la vida colectiva, es decir, que "fija" en la conciencia de ellos, determinado imaginario social o conjunto de ideas sobre la naturaleza humana, sobre las cualidades, habilidades, valores morales, éticos y políticos de los sujetos, sobre los saberes básicos necesarios para poder hacer parte de la sociedad, sobre el individuo, la importancia respectiva de nuestras diferentes facultades, el derecho, el deber, el progreso, la ciencia y el arte; proceso que empieza generalmente con la familia y continúa a través de toda su vida, principalmente, con la educación institucionalizada, aunque actualmente adquieren también mucha importancia los medios masivos de comunicación.

Los diferentes matices de los ideales que se interiorizan construyen significados de la realidad en los sujetos, en conjunto, esquemas de pensamiento, sentimiento, percepción y acción ${ }^{2}$ que estructuran formas determinadas de ser $y$, en general de desenvolverse en la sociedad en la que se encuentran.

De lo expuesto, se puede decir que la educación trasmite y enseña determinados sistemas de significados, además de construir otros (ya que es una práctica y está basada en las relaciones de los individuos), pero sobretodo que su papel es significativo en la legitimación y "normalización" de dichos sistemas representados en saberes, relaciones, practicas, concepciones o nociones, que finalmente se constituyen en verdades, y de esta manera son asumidos como acontecimientos naturales o propios de la condición humana, haciendo más difícil la exploración del pensamiento y la acción en vías diferentes de las que artificialmente se han dado. De esta manera, es adecuado pensar la educación como un escenario fundamental y, por qué no estratégico para la consolidación de "acuerdos", opiniones, fines, objetivos y decisiones de diferente índole, aún más si se condensa en unos tiempos y espacios determinados (escuela), con unos actores educativos (específicos) involucrados y unas intencionalidades reconocidas abiertamente ${ }^{3}$.

\footnotetext{
${ }^{1}$ Estudiante de Licenciatura en Biología. Séptimo Semestre. Universidad Pedagógica Nacional.

2 Bordieu denomina a dicho conjunto habitus.

3 Para Varela (1995) citada por Martínez (3003), esta forma específica de educación se denomina escolarización. “Escolarizar es segregar a los menores de los adultos mediante el procedimiento de alojarlos en lugares ad hoc bajo el cuidado de ciertos sujetos por varias horas al día durante cierta porción del año", p. 2-3.
} 
De manera general, con la institucionalización del quehacer educativo e inherentemente la concentración de todo lo que involucra, se asegura o se aumenta las posibilidades de obtener lo planteado para ella.

Es importante resaltar cómo del mismo seno de la educación "artesanal o intuitiva" emerge un tipo de educación (con determinadas características) que a su vez origina otras formas de subjetividad, es decir, constituye sujetos que antes no se concebían como estudiante/alumno o profesor/maestro; igualmente origina o consolida diferentes saberes como la pedagogía, la didáctica, o los que constituyen en sentido estricto los planes de estudio, con lo que se legitima su importancia, pertinencia y necesidad en la formación de sujetos sociales.

La relevancia del papel de la educación en Colombia es acentuado por el hecho que desde aproximadamente 1870, época de la Reforma Instruccionista, del Decreto Orgánico de la Universidad Nacional y del Decreto Orgánico de la Instrucción Pública se impulsa la educación popular para todos, específicamente la "instrucción primaria popular como una tarea pública nacional, concebida como alfabetización gratuita, obligatoria y universal, garantizada tanto financiera como políticamente por el Estado", (Saldarriaga, 2003). Este acontecimiento evidencia un primer reconocimiento de los alcances de la educación en lo relacionado con la modernización del país.

Al ser declarada abiertamente la trascendencia de la educación, y sobretodo al ser proclamada como un derecho y deber, adquiere aún más valor como escenario estratégico, si se tiene en cuenta que inicialmente la educación era un "privilegio" que sólo unos pocos, las clases sociales con más recursos económicos, podían tener. Con el ideario liberal, de un sistema educativo nacional uniforme, gratuito y laico, se iniciaba por primera vez en el país una campaña masiva de instrucción primaria popular, que abría las puertas de la educación a todos, y al mismo tiempo la posibilidad de mayor cobertura e impacto social de las decisiones que se tomaban en torno a ella.

De igual manera, dicho proceso de modernización dotó (a través de diferentes mecanismos) a la educación de un valor diferente al que se le venía dando, pues ligado a la alfabetización estaba la posibilidad de ejercer el derecho al voto y a su vez de acceder a la condición de ciudadano (Saldarriaga, 2003). El dispositivo discursivo y las acciones concretas emprendidas, generaron toda una serie de nuevos sentidos alrededor de la educación, los actores involucrados y sus responsabilidades, el sujeto y su reconocimiento en la sociedad, la movilización social, las perspectivas, expectativas y necesidades de los sujetos, en otras palabras, de los ideales sociales.

La situación en la que se encuentra la educación, según lo mencionado anteriormente, la convierte desde sus primeros intentos de consolidación como un sistema educativo en campo de confrontación y lucha constante por su dominación. Conflicto (no declarado abiertamente) caracterizado por la participación de distintos actores sociales, económicos y políticos nacionales e internacionales, que con sus intereses - algunas veces diferentes, otras afines- han enmarcado la educación y su configuración en el sistema educativo nacional por constantes 
tensiones y ambigüedades. Algunos autores han expresado que estas mismas condiciones han impedido que se conforme un proyecto educativo nacional, en el sentido estricto de la palabra, es decir, coherente con la complejidad cultural, social, económica y política del país (Martínez ,2003. En Herrera, Pinilla, Infante \& Díaz, 2005).

Como resultado de este contexto, las reconceptualizaciones alrededor de la educación no sólo tienen implicaciones para el campo educativo, sino que sus efectos se difunden en la sociedad, en donde inciden en la configuración de las representaciones sociales alrededor del valor de la educación dentro de ella, además de la función de sus actores directa o indirectamente involucrados como los maestros, el Estado, las instituciones responsables del financiamiento, administración y gestión, afectando otras concepciones como: derecho, deber, servicio, calidad, competencia, cobertura, evaluación, entre otros.

En pocas palabras, las transformaciones de la educación (y por consiguiente del sistema educativo), a través del devenir histórico del país, no solo la han marcado individualmente, sino que además, por ser una práctica cotidiana y "normal", han mediado en la configuración de la sociedad.

Teniendo en cuenta lo anterior, el objetivo del presente escrito es abordar algunas nociones relacionadas con la educación y el quehacer educativo, y en consecuencia con el hombre, la sociedad y la cultura colombiana en relación con las políticas educativas más representativas de los últimos tiempos, sin perder de vista que son consecuencia de prácticas en distintos escenarios cotidianos (económico, el político y social), de su interacción y de la confluencia de unas condiciones y hechos determinados, y que por dicha razón no deberían ser asumidas como verdaderas y naturales. Partiendo de estas perspectivas, es conveniente plantear la siguiente pregunta: ¿Cómo las políticas educativas de los últimos diez años fomentan determinadas nociones y por consiguiente representaciones sociales de la escuela, la educación, la enseñanza, el aprendizaje, la evaluación, el profesor y el estudiante, y cuáles son dichas representaciones?

\section{Políticas Educativas desde los 90's vs. Nociones de la educación y sociedad}

Desde los años 90, aproximadamente, las políticas educativas en Colombia han venido impulsando cambios de mayor trascendencia y repercusiones sociales que los ejecutados en años anteriores, en el sentido de diferenciarse estructuralmente de estos últimos. Partiendo de ésta época, es posible identificar dos momentos diferentes en relación con la formulación de objetivos y el emprendimiento de acciones concretas en el campo de la educación, que van de la mano con un cambio sustancial en la participación de los actores involucrados.

El telón de fondo de esta situación es la carta constitucional de 1990, a partir de la cual se justifican y legitiman las posteriores leyes y reformas educativas. Dicha constitución expresa propuestas tendientes a la defensa de la educación pública, además de concepciones economicistas apoyadas en el enfoque neoliberal, que ponen en riesgo la existencia de la educación pública. Esta característica es consecuencia de la confluencia de fuerzas políticas y sociales (con intereses disímiles y contradictorios) en su elaboración (Herrera et al., 2005). 
Para Miguel Ángel Urrego, la constitución del 91 “contiene dos visiones de nación que resultan contradictorias: por un lado se establece el principio multiétnico y multicultural, por otro se consagra el neoliberalismo como modelo económico de la nación, lo cual amenaza diluir los frágiles hilos del tejido social y la simbología nacional"' (Urrego, 1998. En Herrera et al., 2005).

Cabe aclarar, que diferentes procesos adelantados en el plano internacional, como el denominado "Consenso de Washington", en el que se definen y establecen un conjunto de "recomendaciones" de política económica destinado a los países latinoamericanos con el objetivo de impulsar su crecimiento, tuvo en aquel momento y tiene en la actualidad un fuerte impacto en las reformas constitucionales, puesto que "Washington" era un complejo políticoeconómico- intelectual constituido, entre otros, por organismos financieros internacionales como el FMI y el BM y la adherencia a este listado y a las posteriores modificaciones legislativas y administrativas se convierten, antes y ahora, en un condicionamiento para los desembolsos de recursos monetarios a los estados por parte de dichas organizaciones.

Así mismo, los procesos de internacionalización y modernización permitieron a los intereses mundiales la introducción y consolidación paulatina de las políticas educativas más representativas de los últimos años.

A partir de las disposiciones educativas de la Constitución del 91, reglamentadas a través de la Ley General de Educación del 94, algunas de las reformas importantes son las hechas a los lineamientos generales de procesos curriculares emitidos por dicha ley, modificados en 1996 con la Resolución 2343 al adicionar los indicadores de logros y en 1998 con la aparición de Lineamientos curriculares por áreas. En el 2001 con la ley 715 se introducen parámetros técnicos para la prestación del servicio educativo estatal, estándares y tasas de asignación de personal, teniendo en cuenta las particularidades de cada región y, finalmente en el 2002, el MEN adopta unos estándares provisionales en áreas como matemáticas, lengua castellana, ciencias naturales y educación ambiental (Bustamante, 2003).

Dichas reformas educativas, que no se encuentran aisladas, se fundamentan en determinada perspectiva de la realidad (sustentada en un modelo o teoría "macro"), a la vez que la legitiman. Esta relación se encuentra mediada por las "políticas educativas", basadas en dos estructuras principales: el dispositivo discursivo y las acciones concretas o programas (Martínez, 2003).

Desde Foucault, el discurso es "un conjunto de hechos lingüísticos, pero especialmente estratégicos y polémicos", un juego de palabras estructurado y organizado con una intencionalidad, de tal forma que se involucra en los diferentes escenarios como medio fundamental de "lucha, de acción y reacción, de pregunta y respuesta, de dominación y retracción". El dispositivo discursivo de las nuevas políticas, correspondientes al modelo neoliberal, tendrían la finalidad de construir todo un campo semántico que dé significado y legitime las acciones basadas en él. En este sentido, el discurso y las acciones concretas son inter-dependientes: el primero configura y construye una realidad que posibilite el desarrollo de los programas, y estos últimos son la razón de ser, o mejor el "lugar" que engendra el 
discurso.

El discurso del modelo neoliberal (base de las actuales políticas y reformas) ha modificado los términos de los problemas ${ }^{4}$ de la educación (ha cambiado los problemas de la educación basados en diferentes representaciones de la realidad), y así mismo sus esquemas de solución. Esto, que según González (2005) se denomina políticas "educativas", ha conllevado a la transformación de otras nociones, que terminan por aportar al fortalecimiento de los acuerdos, opiniones o decisiones que generan dichas políticas.

Los componentes del discurso educativo en los procesos de las reformas educativas de Colombia, y en las demás naciones latinoamericanas, están basados principalmente en la calidad y la equidad (Martínez, 2003). La primera como medida frente al desmejoramiento de la educación, ocasionado por la masificación de los sistemas educativos, incluido en el ideario liberal, (Herrera, et al., 2005) y la segunda como posibilidad que se le abre a toda la población de acceder al goce de unos derechos- o mejor ¿servicios?- según las condiciones de cada uno.

A partir de la formulación de la crisis de la educación en términos de eficacia, eficiencia y productividad, desde la perspectiva de los actores internacionales económicos (que ganan una participación definitiva en la formulación de políticas educativas, ocasionando a su vez que las funciones del Estado desde el ideario liberal cambien) se comienza la consolidación de las nuevas representaciones educativas y sociales.

Junto al dispositivo discursivo, se encuentra las acciones concretas, representadas en elementos como los estándares, mecanismos de evaluación y acreditación, parámetros técnicos para la prestación del servicio educativo estatal, tasas de asignación de personal y profesionalización docente, nuevos cuerpos normativos, renovación curricular, nuevas formas de gestión del sistema educativo y la introducción de las nuevas tecnologías (Martínez, 2003). Además de estrategias de nivel global dirigidas a todos los escenarios de la vida, expuestas por Angulo Rasco de la siguiente manera: “a) El monetarismo: reforzamiento de las fuerzas de mercado libre y de la economía de oferta. b) Políticas de privatización de los servicios públicos. c) El reforzamiento y extensión de la ideología social del mercado, es decir, de la lección y libertad individual, la defensa de la "privacidad" y de la conversión del ciudadano en cliente. d) Los cambios, algunas veces radicales, en la estructura del papel del Estado" (Angulo, 2002. En Herrera et al., 2005). Con la puesta en vigencia de estas reformas, políticas educativas, discursos, estrategias y elementos se puede evidenciar los cambios en el escenario educativo y social (Herrera, et al., 2005).

Las reformas educativas y la educación, anteriormente estaban dirigidas a la "consolidación de sistemas educativos nacionales, en la perspectiva de contribuir a la construcción de culturas políticas acordes con el ideario de las sociedades democráticas" (Herrera, et al., 2005), diferente a las últimas, que más bien están orientadas por la lógica del mercado, por la demanda de intereses económicos de instituciones privadas, y en general, por patrones productivistas y empresariales, reduciendo tanto la participación administrativa y financiera del Estado, como

4 "Los problemas son elaboraciones de los hombres. Siempre existen concepciones múltiples y no hay problemas definidos de manera única”. WILDAVSKY (1979) citado en GONZÁLEZ (2005)), p. 63. 
la complejidad del acto educativo.

En el primer aspecto, las prioridades de la educación son dadas por el sector privado, de tal manera que el Estado pierde el control en la regulación del sistema educativo, al mismo tiempo que cede su financiación a este tipo de entidades, ya que uno de los ejes del neoliberalismo es la privatización de todas las formas de vida pública.

Dado que lo público hace referencia a algo de carácter universal y que la educación pública, desde el ideario liberal, debe estar en igualdad de oportunidades para toda la población y es responsabilidad del Estado (en otras palabras lo público como sinónimo de estatal), la privatización obliga a que las funciones del Estado, en cuanto a la financiación y asignación de recursos, sea menor, delegada a los departamentos y municipios o a las mismas instituciones educativas (autofinanciaciación). Situación que está ligada al desplazamiento inicial del concepto cobertura, ya no referido a la atención universalizada sino focalizada en ciertos sectores (los más pobres de los más necesitados), que indudablemente, deja por fuera a las clases medias.

Igualmente, esta privatización conlleva a una resignificación de lo público, asumido no sólo como la gestión exclusiva del Estado, sino también la participación de empresas privadas, encargadas de la prestación de servicios.

Dicho reajuste financiero se encuentra relacionado con la austeridad y eficiencia, que tiene como objetivo cumplir los compromisos económicos con organizaciones financieras internacionales. También es importante mencionar, que al perderse el carácter público de la educación se afecta el concepto de ciudadano como sujeto social y político participativo frente al sistema y así mismo a una cultura política que reconozca todos los actores sociales, esto en la medida que lo convierte en cliente y consumista, aspecto regulado a su vez por el mecanismo de oferta y demanda.

Esta misma crisis que afecta la educación pública debe entenderse como un atentado a la democracia y al real reconocimiento de todos los sujetos de la sociedad (Giroux, 2002. En Herrera, et al., 2005).

\section{El acto educativo y el maestro}

En cuanto a la incidencia en el acto educativo, es evidente principalmente, en dos aspectos: en la enseñanza, ya que al establecer las competencias que todos los niños deben haber adquirido a través del proceso, por ejemplo con los estándares, no se tiene en cuenta las condiciones y las prioridades propias de la institución educativa, que en algún momento pueden obstaculizar la consecución de dichos criterios, repercutiendo en otras cuestiones como la asignación de recursos, o la estabilidad laboral de los docentes. Además, en muchas ocasiones dichos elementos son asumidos como contenidos escolares por los maestros, lo que puede fomentar su desinterés o tranquilidad hacia el oficio docente, transformando la perspectiva de la enseñanza a una mera transmisión de conocimientos o destrezas, y de su fundamental papel como sujeto social en la transformación de la sociedad. Dicha concepción conlleva a cuestionar la importancia del maestro en la sociedad, y que su labor pueda ser reemplazada por las 
nuevas tecnologías.

Inherente a este último punto, se encuentran los cambios que pueden sufrir los dos saberes base en la formación de cualquier maestro, la pedagogía y la didáctica, y es así, puesto que éstos son los que le dan verdadero sentido a la enseñanza y a la educación, al orientarla y organizarla alrededor de unos procesos intencionales.

De esta manera, si asumimos la pedagogía como "la reflexión de la educación, del cómo, por qué y hacia dónde" y la didáctica como "el saber que tematiza el proceso de instrucción o enseñanza y orienta sus métodos, su estrategias, su eficiencia.., orientada por un pensamiento pedagógico" (Ruíz \& Ramírez, 2001) es innegable pensar en su importancia para la reflexión crítica y la investigación en el salón de clase, convirtiéndolas en herramientas fundamentales para la retroalimentación de la enseñanza y en general, del proceso educativo.

De lo anterior se puede deducir que si se desvirtúa la imagen elemental de dichos saberes por parte de los maestros, la enseñanza perdería su valor formativo y crítico para constituirse en simplemente la utilización de unos recursos con el propósito de transmitir criterios ya estipulados.

En relación con los estándares y las pruebas internacionales, está el concepto de calidad, que contrario a lo que venía siendo en las décadas de los 60 y 70, al responder a las exigencias y necesidades de los maestros y de las comunidades, ahora se considera como una herramienta de racionalización de la inversión y optimización de los resultados (Saviani, 1998. En Herrera, et al., 2005).

Sumado a los anteriores conceptos, se encuentra el de evaluación, un instrumento neoliberal relacionado con el logro de los estándares planteados, herramientas predecibles y eficientistas que no aportan un verdadero conocimiento del desempeño de la escuela. Estas mismas políticas de evaluación han aportado para que se incluya la profesionalización del magisterio o racionalización de las plantas de personal docente, es decir, que contribuyan a la organización del trabajo de manera que aumente los rendimientos o reduzca los costos con el mínimo de esfuerzo que se requiere para poner en marcha el "sistema empresarial educativo", lo que a su vez reduce a la pedagogía a una técnica instrumental y conductista que tiene como finalidad la trasmisión de unas habilidades y conocimientos mínimos que le permitan al sujeto desempeñarse en determinado rol en la esfera laboral, según a los estándares laborales, es decir que sea competente.

Desde esta perspectiva, "la escuela tiene por función, en la perspectiva de los hombres de negocios, la transmisión de ciertas habilidades y competencias necesarias para que las personas se desempeñen competitivamente en un mercado de trabajo altamente selectivo y cada vez más restringido. La educación escolar debe garantizar las funciones de selección, clasificación y jerarquización de los postulantes a los futuros empleos" (Gentili, 1997. En Herrera, et al., 2005). 
Igualmente, el estudiante y los objetivos planteados para ellos, lo reducen a un sujeto que simplemente adquiere habilidades y competencias, sin posibilidades de participación activa en su propio proceso de aprendizaje, que a propósito no es asumido como la capacidad general de aprender sino como mera adquisición de ciertas competencias determinadas por sus resultados efectivos. De esta manera, con la trasmisión y adquisición de unos determinados sistemas de significados en la escuela, se pretende formar personas que en etapa laboral sean aptas para la producción, o en la infancia y vejez se conviertan en clientes y demandantes de una producción económica.

El aprovechamiento de toda la población, sin duda alguna, incluye a los pueblos indígenas y la multiculturalidad de Colombia, ya que con la introducción de todos estos elementos de las nuevas políticas educativas, en especial los estándares, se obliga paulatinamente a que renuncien a las construcciones que han hecho del mundo y que le dan significado a la realidad desde sus perspectivas, por ejemplo, cuando quieran ingresar a otros contextos o desenvolverse fuera de sus comunidades.

Con la introducción de las nuevas políticas, no sólo en Colombia, sino en América Latina en general, se tiende progresivamente a la homogenización de la diversidad cultural y social que tanto caracteriza a esta parte del continente, encaminadas a la producción en masa y a la satisfacción de deseos consumistas.

De cara a este panorama educativo, no está de más decir, que nos compete a todos interesarnos por su situación e interferir en esos acontecimientos cotidianos, y por qué no "normales" que pasan inadvertidos ante nuestros ojos y oídos (cuando suceden), pero que tienen profundas repercusiones en la definición de la educación y la sociedad que queremos. Todavía más cuando somos los maestros los que estamos más directamente involucrados y en contacto diario con el quehacer educativo, recae en nuestro oficio la generación de este tipo de "conciencia".

La indagación y reflexión del presente de la educación de Colombia, que ha interesado tanto en los últimos tiempos a todo tipo de estudiosos, debe ser una fuente primaria (pero no la única) para que nosotros, los maestros en formación, permanezcamos en constantes cuestionamientos de lo que nos rige, de lo que afecta de manera directa el proyecto de vida que hemos escogido. Teniendo como apoyo las tensiones actuales a nivel político, económico y social, resultado de un devenir histórico marcado por confrontaciones ideológicas tanto nacionales como internacionales, podemos acercarnos con una mirada más amplia a dicha situación y a las acciones que en la dimensión pedagógica, educativa, política y de manera general en el quehacer cotidiano en las escuelas, debemos y podemos emprender, posibilitando así practicas y representaciones políticas y sociales alternativas a las que, a la luz de modelos neoliberales, se han presentado en los últimos años.

En esta medida una reflexión de este tipo aporta elementos valiosos, especialmente en la formación inicial de maestros, ya que expone la situación por la que atraviesa actualmente la 
educación y cómo esto contribuye en la formación de una sociedad con determinadas características, muy en consonancia con la de las hegemonías y sectores dominantes, lo que hace pensar en el papel trascendental de la educación, y especialmente de los maestros, para hacer resistencia crítica y transformadora.

\section{BIBLIOGRAFÍA}

-Angulo, M.A. (1998, marzo). Mitos fundacionales, reforma política y Nación en Colombia. En Nomadas. Santa Fé de Bogotá. Universidad Central. N 8.

-Bustamante, G. (2003, enero-junio). Estándares curriculares y autonomía. En Revista Colombiana de Educación. $\mathrm{N}^{\circ} 44$.

-Gentili, P. (1997) .Adiós a la escuela pública; el desorden neoliberal, la violencia del mercado y el destino de la educación de las mayorías. En Cultura, política y currículo: ensayos sobre la crisis de la escuela pública. Buenos Aires.

.Durkheim, E. (1970). La educación como proceso socializador: función homogeneizadora y función diferenciadora. Buenos Aires.

-Giroux, H. (2002) Pedagogía pública y política de la resistencia. Notas para una teoría crítica de la lucha educativa. En Opciones pedagógicas. Bogotá. $\mathrm{N}^{\circ} 25$.

.González, A.M. (Diciembre 2005). Poder, política y políticas modos de empoderamiento del maestro. En Educación y ciudad. N 9.

.Focault, M. (1973).La verdad y las formas jurídicas. Primera de Cinco conferencias dictadas en la Universidad de Río de Janeiro,

.Herrera, M.C, Pinilla, A., Infante, R. \& Díaz, C. (2005).Educación pública y cultura política en Colombia. En: La construcción de cultura política en Colombia Proyectos hegemónicos y resistencias culturales. Universidad Pedagógica Nacional. Bogotá.

-Martínez, A. (2003, marzo).De la escuela expansiva a la escuela competitiva. Dos modos de modernización en América Latina. Ponencia presentada en el IV Congreso Iberoamericano de Historia de la Educación. México, 19-24.

.(2003). Currículo y Modernización. Cuatro décadas de educación en Colombia. Segunda edición. Universidad Pedagógica Nacional- Grupo Historia de la Práctica Pedagógica. Magisterio. Bogotá.

- Ruíz, A. \& Ramírez, J. (2001). Enfoques y tendencias pedagógicas contemporáneas. Noviembre.

Saldarriaga, O. (2003). Del oficio de ser maestro. Prácticas y teorías de la pedagogía moderna en Colombia. Colección pedagogía e historia. Cooperativa editorial Magisterio. Bogotá DC.

-Saviani. (1998). La productividad de la escuela, un re-examen de las relaciones entre educación y la estructura económico-social capitalista. Buenos Aires. 